\title{
Liposomes - bio-inspired nano-containers for physically triggered targeted drug delivery
}

\author{
Sofiya Matviykiviv, Marzia Buscema ${ }^{\mathrm{a}}$, Tamás Mészáros ${ }^{\mathrm{b}, \mathrm{c}}$, Gabriela Gerganova $^{\mathrm{a}}$, Thomas Pfohl ${ }^{\mathrm{a}}$, \\ Andreas Zumbühl ${ }^{\mathrm{d}}$, János Szebeni ${ }^{\mathrm{b}, \mathrm{c}}$ and Bert Müller ${ }^{\mathrm{a}}$ \\ ${ }^{a}$ Biomaterials Science Center, University of Basel, Gewerbestrasse 14, 4123 Allschwil, Switzerland; \\ ${ }^{\mathrm{b}}$ Nanomedicine Research and Education Center, Semmelweis University, Nagyvárad tér 4, 1089 \\ Budapest, Hungary; 'SeroScience Ltd., Nagyvárad tér 4, 1089 Budapest, Hungary; \\ ${ }^{\mathrm{d}}$ Department of Chemistry, University of Fribourg, Chemin du Musée 9, 1700 Fribourg, Switzerland
}

\begin{abstract}
For natural scientists and engineers, learning from nature has tradition and is often driven by bio-inspired processes and materials. For example, engineers have designed multifunctional materials with hierarchical structures. Lipid bilayers, the principal components of cell membranes, can form vesicles, termed liposomes. Such liposomes are usually recognized as foreign by the immune system of a patient, which makes it challenging to use liposomes as containers for targeted drug delivery. There are, however, promising non-spherical, mechano-sensitive, artificial liposomes about $100 \mathrm{~nm}$ in diameter, which were recently identified. These bio-inspired containers offer a wide range of applications. In particular, the targeted release at critically stenosed arteries formed as a result of atherosclerosis significantly reduces the undesired side effects such as a drop of blood pressure. It is well known that FDA-approved liposomal drugs, currently on the market, often induce adverse immune responses. Therefore, to exclude the hypersensitivity of the recently discovered mechano-sensitive liposomes, we have performed in vitro complement activation experiments and related animal studies with pigs. Recently, it has been shown that the drug-free Pad-PC-Pad liposomes surprisingly lack any complement activation. In this study, we demonstrate that nitroglycerin-loaded liposomes with relevant human therapeutic dosage exhibit low complement activation compared to the FDA-approved phospholipid drugs, including Abelcet. Furthermore, the liposomal suspensions applied are stable for a period of more than two months. Consequently, the non-spherical liposomes of nanometer size we have developed are promising containers for physically triggered, targeted drug delivery.
\end{abstract}

Keywords: Nanotechnology, targeted drug delivery, atherosclerosis, biomimetic, liposome, shear stress, hypersensitivity, complement activation.

\section{INTRODUCTION}

Nanomedicine is at the frontline of nowadays therapy for targeted drug delivery and the global nanomedicine market continues to grow. It is expected to reach $\$ 528$ billion by 2019 [1]. The goal of such transporting system is to safely assist the delivery of a pharmaceutical compound to a specific targeted within the body, increasing the therapeutic index of the drug and decreasing acute side effects.

Nature has inspired most of the successfully introduced technologies, that are used in biomedical applications. Biomimetical systems were developed, based on the way how the natural systems are constructed. For instance, biological membranes, representing highly complex and dynamic structures, are mimicked in lipid self-assembly liposomes, closed, spherical vesicles, trapping an aqueous solution into inner environment (see Figure 1).

The spontaneous liposome formation is initiated when phospholipids, with two hydrophobic chains and a hydrophilic polar head group, are exposed to an aqueous environment. This configuration is energetically favored. Liposomes were firstly described by Bangham and Horne in 1964 [2]. Inspired by this architecture, liposomes appeared as a suitable concept for the development of drug delivery systems [3-5].

*bert.mueller@unibas.ch; phone +41 6120754 30; fax +41 6120754 99; www.bmc.unibas.ch

Bioinspiration, Biomimetics, and Bioreplication 2017, edited by Mato Knez, Akhlesh Lakhtakia, Raúl J. Martín-Palma Proc. of SPIE Vol. 10162, 101620A · @ 2017 SPIE · CCC code: 0277-786X/17/\$18 · doi: 10.1117/12.2258378 


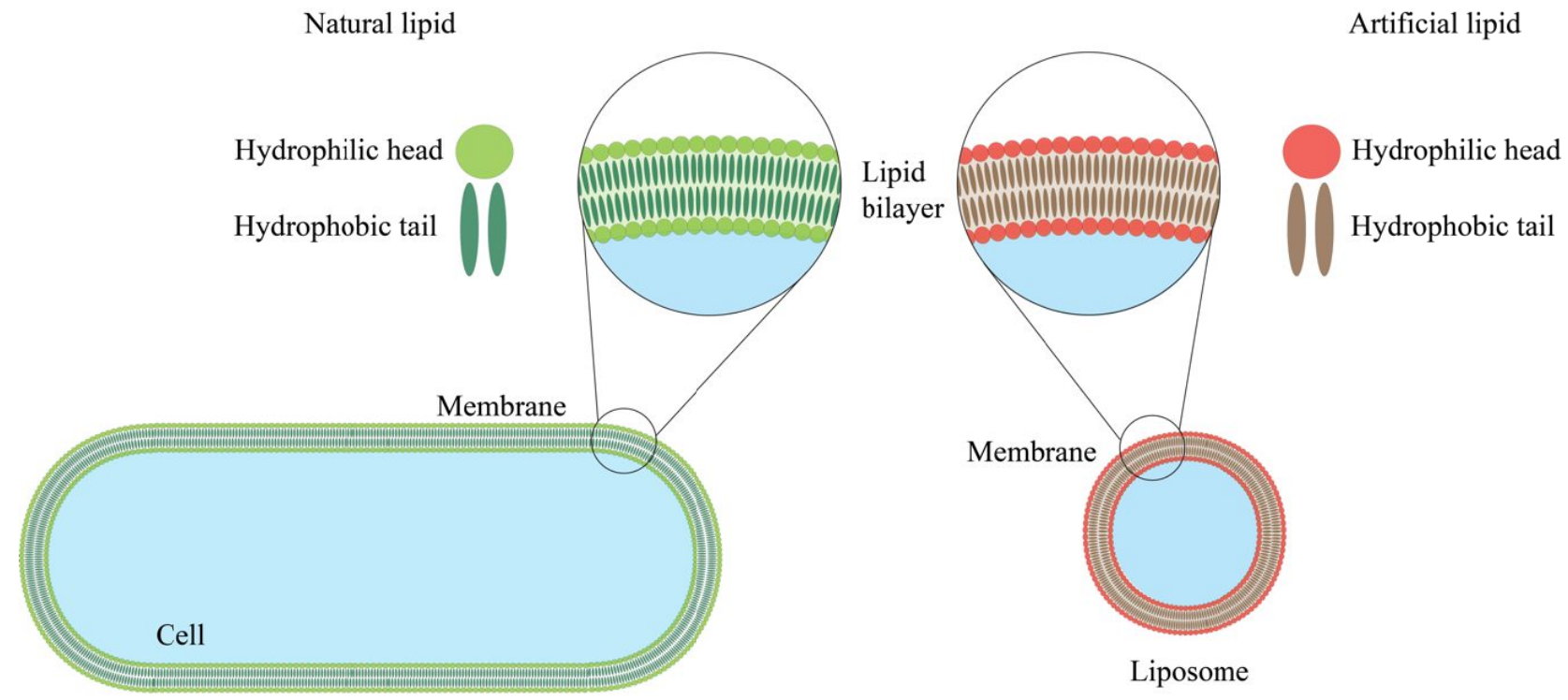

Figure 1. Schematic representation of cell and liposomal lipid bilayer structures. The size of a cell is roughly 100 times larger than the diameter of a liposome. However, in both cases the membrane thickness is the same, about 4-5 nm.

The types of phospholipids used in the fabrication of liposomes are crucial in determining the liposomal properties. Expanding into the non-natural chemical space, we have synthetized artificial 1,3-diamidophospholipids that form liposomes with a faceted morphology [6]. These nanometer-sized, lentil-shaped liposomes are sensitive to the mechanical stress, and have the unique property to release their cargo upon shaking [7]. It makes them a favorable candidate for delivery of vasodilators to constricted arteries, where the liposomes are exposed to an increased average wall shear stress, similar to shaking. Significant changes in the increase of shear stresses between healthy and constricted regions of arteries, are the result of the narrowing of the arterial blood vessels due to plaque formation related to the well-known disease atherosclerosis [8]. 17.5 million people die each year from cardiovascular diseases (CVDs), that is around $31 \%$ of all deaths worldwide [9]. These numbers obviously support the need of further studies, especially for the first line treatment of CVDs. Pre-hospital treatment of acute myocardial ischemia comprises bolus administration of nitroglycerin to dilate blood vessels [10]. When applied intravenously it may cause rapid systemic drop down of the blood pressure and, as a result, reduced blood perfusion, which can be lethal in severe cases. Therefore, its combination with nanostructured liposomes may improve the local efficacy of the encapsulated nitroglycerin and mitigate negative side effects.

Despite the successful clinical application of nanomedicines as a controlled drug delivery systems [11], the intravenously injected liposomal drugs are generally recognized by components of the innate immune system as foreign particles. This leads to the activation of a biochemical cascade of the innate immune system, termed complement activation (see Figure 2), resulting in severe hypersensitivity reactions (HSRs) [12]. There are three pathways that initiates complement activation and results in the formation of the membrane attack complex (MAC): the classical, lectin and alternative pathways. The classical pathway leads to complement activation via the binding of antibodies to pathogen surface antigens. This involves the recruitment of $\mathrm{C} 1$ complex, which subsequently activates its natural substrate $\mathrm{C} 2$ and $\mathrm{C} 4$. Mannose-binding lectin (MBL) protein binds to carbohydrate ligands on the pathogen surface, and together with MASP (Mannose-binding lectin-Associated Serine Proteases) they form a complex that proceeds to the cleavage of C2 and C4. The classical and lectin pathways converge at an early stage of the cascade. They trigger the formation of C3-convertase that continuously hydrolyzes $\mathrm{C} 3$, and results in amplification of the complement cascade signal. The possibility to study the role of classical and lectin pathways in complement activation, relies on the detection of the C4d protein. C4d is a split product of $\mathrm{C} 4$ activation, and is mainly interpreted as a trace of classical pathway activation, however, it is also derived from the lectin pathway [13]. An alternative pathway initiates when a cleaved C3b protein directly binds to the antigen, such as endotoxin, which is found in the outer membrane of bacteria, and elicit strong immune responses. Along the activation cascade, the factor B becomes cleaved, resulting in the formation of the split product Bb, which can be used as a marker for detection of the alternative pathway. The classical and lectin complement activation pathways converge with the alternative at the level of $\mathrm{C} 3$ convertase and proceeds into further cleavage of $\mathrm{C} 5$ with the subsequent 
formation of the C5b-9 membrane attack complex on complement-activating surfaces as represented in Figure 2. It causes direct cell injury by the formation of transmembrane channels, which disrupt the cell membrane, leading to cell lysis and death. If the target membrane is absent, the C5b-9 complex binds to vitronectin (a regulatory $\mathrm{S}$ protein). Therefore, liposome-mediated complement activation in human serum was first monitored by measuring the generation of SC5b-9 complex, also termed Terminal Complement Complex (TCC), as a marker for the activation of the whole complement cascade [14].

We hypothesize that artificial liposomes of nanometer size exist that do not show any complement activation for the human therapeutic dose of encapsulated drug. In case of an at least partial approval, a wide variety of therapeutic applications can be envisioned.

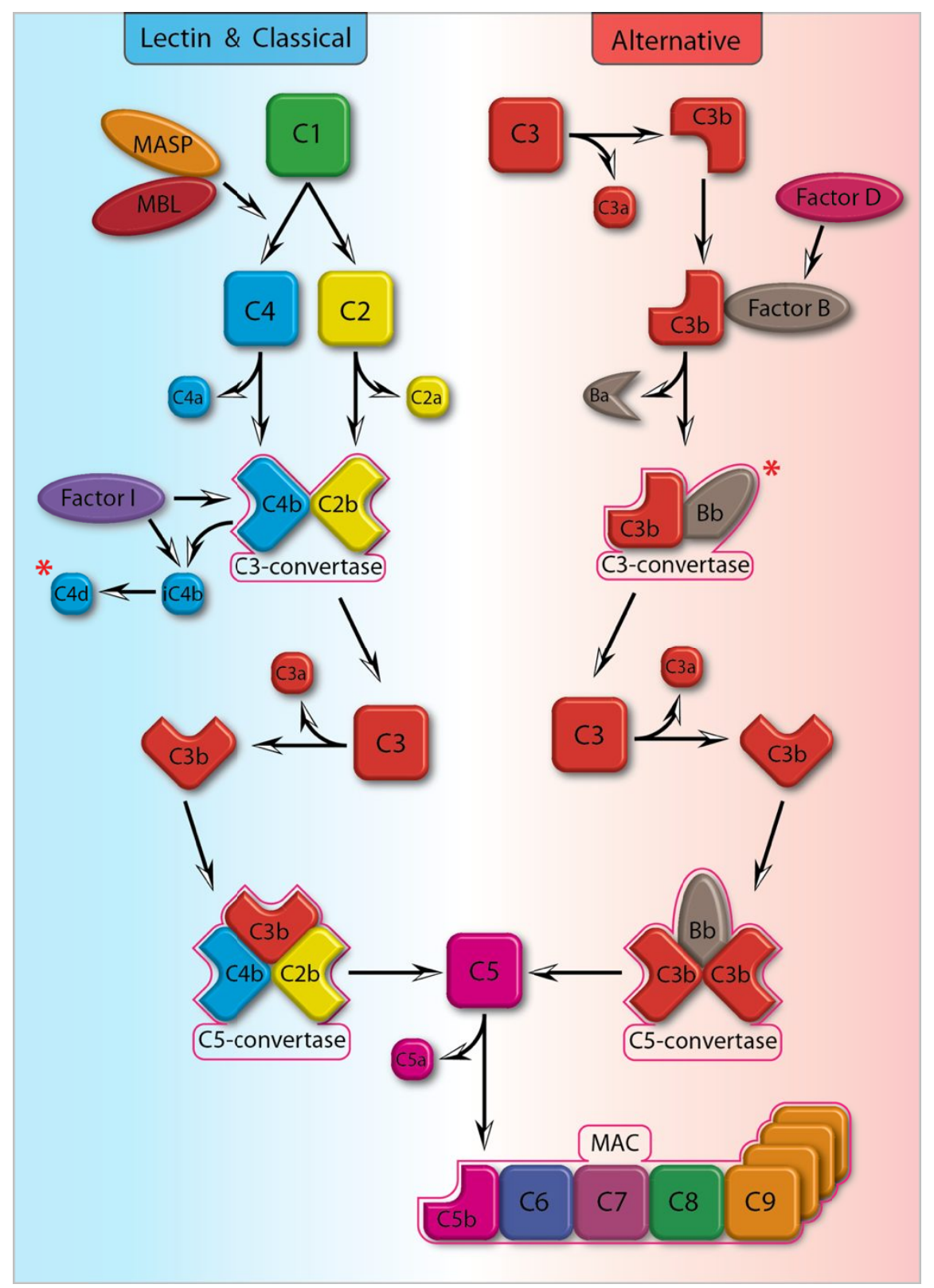

Figure 2. Schematic representation of the complement system cascade classified according to the classical, lectin, and alternative pathways. 


\section{MATERIALS AND METHODS}

\subsection{Materials for in vitro ELISA assay}

Pad-PC-Pad (1,3-palmitoylamido-1,3-deoxy-sn-glycero-2-phosphatidylcholine) has been synthetized following the previously reported protocol [6, 7]. DSPE-PEG 2000 (1,2-distearoyl-sn-glycero-3-phosphoethanolamine-N[amino(polyethylene glycol)-2000]) was a generous gift from Lipoid GmbH (Germany). Commercial nitroglycerin (NTG) solution "Perlinganit" was purchased from UCB-Pharma AG (Switzerland), Zymosan - from Sigma-Aldrich Ltd. (Hungary) and saline solution $(0.9 \%, 308 \mathrm{mOsm} / \mathrm{L})$ - from Teva Pharmaceuticals Zrt (Hungary). Doxil and Abelcet were used as purchased by the Nanomedicine Research and Education Center, Semmelweis University (Hungary). Human serum and pig plasma samples were obtained from healthy donors at the Semmelweis University, according to an approved phlebotomy protocol. Until usage, the samples were stored at a temperature of $-80{ }^{\circ} \mathrm{C}$. For the determination of classical, alternative pathways and terminal complement complex the following ELISA kits were used: MicroVue C4d, Bb and SC5b-9 Plus. ELISA kits were purchased from Quidel Corp. (San Diego, CA, USA).

\subsection{Preparation of shear stress sensitive Pad-PC-Pad liposomes}

Three liposomal formulations were prepared, namely A (Pad-PC-Pad/DSPE-PEG 2000 ), B (Pad-PC-Pad/DSPE-PEG 2000 ) and C (Pad-PC-Pad). Liposomes were prepared from Pad-PC-Pad and DSPE-PEG ${ }_{2000}$ phospholipids in compliance with a previously reported protocol [7], using the thin film method [15, 16]. Briefly, the lyophilized phospholipids were dissolved in chloroform, in appropriate molar ratios: A - 95:5, B - 95:5, C - 100 mol\%. The initial phospholipid concentration was $10 \mathrm{mg} / \mathrm{mL}$. Then, the solvent was removed by rotatory evaporation under reduced pressure, followed by high-vacuum drying overnight. Three lipid films were then hydrated for a period of 30 minutes each at the temperature of $60{ }^{\circ} \mathrm{C}$, with the corresponding buffer: A - 0.1 vol \% NTG, B - 0.9 vol \% saline, C - 0.1 vol \% NTG. Afterwards, the liposomal suspensions were subjected to ten freeze-thaw cycles: frozen in liquid nitrogen and thawed in a $60{ }^{\circ} \mathrm{C}$ water bath. The liposomes were sized by multiple extrusions through Nuclepore ${ }^{\mathrm{TM}}$ track-etched polycarbonate membrane filters (Whatman, GE Healthcare Life Sciences, UK) of pore sizes of 400, 200, and 100 nm diameter pore sizes, until their diameter distribution became almost monodisperse. Multiple extrusions were performed using a barrel extruder Liposofast LF-50 (Avestin Inc., Canada) at 50 bar $\mathrm{N}_{2}$ pressure and a temperature of $65{ }^{\circ} \mathrm{C}$. The samples loaded with Perlinganit were purified from excess of external NTG by buffer exchange using PD-10 desalting columns (GE Healthcare Bio-Sciences AB, Sweden), with an exclusion limit of 5,000 Da, following the manufacturer's gravity protocol. The samples were stored at room temperature until use. To reach the human therapeutic dose (HTD) of encapsulated nitroglycerin each liposomal formulation $(\mathrm{A}, \mathrm{B}, \mathrm{C})$ was diluted ten times $\left(\mathrm{A}^{*}, \mathrm{~B}^{*}, \mathrm{C}^{*}\right)$ before in vitro testing (cf. section 3.2).

\subsection{Characterization of lentil-shaped Pad-PC-Pad liposomes}

\section{Measurement of size and size distribution}

The size and size distribution (polydispersity index, PDI) of the obtained liposomes was determined by triplicate measurements of each sample with the dynamic light scattering (DLS) technique using a DelsaNano $\mathrm{C}$ instrument (Beckman Coulter, USA) at a temperature of $25^{\circ} \mathrm{C}$. Measurements were performed on freshly prepared samples. The average diameter of the liposomes within each sample and standard deviations were calculated using the Smoluchowski model (see Table 1 below). The liposome stability over time was tested after 10, 30 and 60 days subsequent to preparation (see Figure 4 below).

\section{Measurement of zeta potential}

The zeta $(\zeta)$ potential of the samples was measured using the laser Doppler electrophoresis technique on a Zetasizer Nano-ZS (Malvern Instruments Ltd., UK). Liposomes samples were freshly prepared and measured with the following specifications: $60 \mathrm{~s}$ sampling time; $0.8872 \mathrm{cP}$ medium viscosity; 1.33 refractive index; $173^{\circ}$ scattering angle, using noninvasive backscatter optics; $25^{\circ} \mathrm{C}$ temperature (see Table 1 below). Data were analyzed using the Zetasizer software supplemented with the device. The zeta potential values were calculated using the Helmholtz-Smoluchowski's equation.

Measurement of size and morphology

Liposomes size and morphology were analyzed using transmission electron microscopy (TEM), using CM-100 electron microscope (Philips, Eindhoven, Netherlands), operating at an accelerating voltage of $80 \mathrm{kV}$. Samples were prepared by liposome deposition onto a 400-mesh carbon/palladium-coated copper grid (Polysciences Inc., Eppelheim, Germany), 
which was exposed for $10 \mathrm{~s}$ to plasma treatment prior to exploitation. Liposomal samples were negatively stained using a $2 \%$ uranylacetate solution (Sigma-Aldrich, Buchs, Switzerland), and left to dry overnight at room temperature.

\section{Determination of phospholipid concentration}

The phospholipid content of the samples was determined by three independent measurements of each sample with the phosphate test 2.0 [17]. Briefly, $20 \mu \mathrm{L}$ of a liposomal suspension was diluted 1:10 with ultrapure water $(18.2 \mathrm{M} \Omega \mathrm{cm})$ in a $0.5-2 \mathrm{~mL}$ microwave vial (Biotage, Sweden). Then $500 \mu \mathrm{L}$ of a mixture of nitric acid and sulfuric acid (3:1 v/v) were added. The vial was sealed and heated for a period of 20 minutes in a microwave (Biotage Initiator, Sweden) to a temperature of $180^{\circ} \mathrm{C}$. After the vial was cooled down, the following solutions were added in sequence: water $(2.3 \mathrm{~mL})$, ammonium metavanadate and ammonium heptamolybdate coloring agent $(1 \mathrm{~mL})$, and sodium hydroxide solution $(1 \mathrm{~mL}$, $10 \mathrm{M}$ ). After a duration of 10 minutes the solution was pipetted into a 96-well plate (Nunc-Immuno plate F96 Polysorb, Denmark). Three repeated measurements with eight replicates were performed. The dilution factor specific to the 96well plate was 33.3. The absorbance was measured at a wavelength of $405 \mathrm{~nm}$ using a MultiskanFC 96 plate reader (Thermo Scientific, USA).

\subsection{In vitro complement ELISA assays for human serum samples}

Six human sera were thawed to a temperature of $4{ }^{\circ} \mathrm{C}$ and mixed with the following samples: three liposomal suspensions $(A, B, C)$ and their 10-times diluted versions $\left(A^{*}, B^{*}, C^{*}\right)$, negative controls (saline and nitroglycerin), FDA-approved liposomal suspension (Doxil) and lipid complex (Abelcet), and the positive control (Zymosan). Eleven samples were incubated with a serum from each of the six donors at a temperature of $37.1^{\circ} \mathrm{C}$, in a ratio $3: 1$. A stock solution of each sample was prepared. After incubation period of 40 minutes the reaction was terminated by adding $10 \mathrm{mM}$ ethylenediaminetetraacetic acid (EDTA), as stop solution. 0.5 M EDTA was diluted with specimen diluent provided by the kit to obtain a concentration of $10 \mathrm{mM}$. This 20-times sample dilution, is needed as a minimum dilution required to detect the optical density (OD). In order to obtain appropriate OD detection range for each of the samples, we prepared additional dilutions of some liposomal suspensions and positive control: SC5b-9 kit: A - 12x, B - 10x, Doxil 1.5x, Abelcet - 10x, Zymosan - 25x; C4d kit: Zymosan - 2x; Bb kit: Abelcet - 4x; Zymosan - 6x. The negative control stayed undiluted. During the whole experiment the sera were kept in an ice bath to avoid any spontaneous complement activation. Figure 3 schematically represents the sample preparation steps for the ELISA assay.

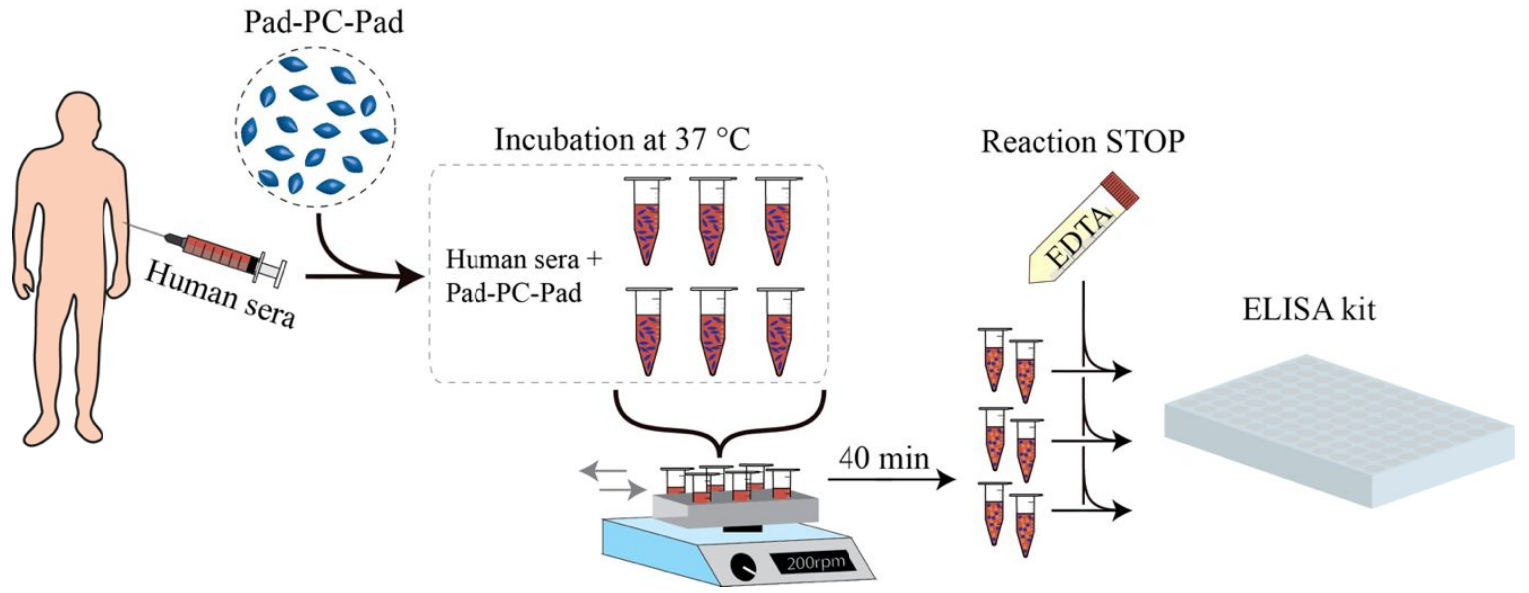

Figure 3. Schematic representation of sample preparation for in vitro complement assays of human sera.

\section{Classical and alternative pathways of complement activation}

To detect the activation of classical and alternative pathways, the levels of the $\mathrm{C} 4 \mathrm{~d}$ and $\mathrm{Bb}$ proteins were tested by MicroVue $\mathrm{C} 4 \mathrm{~d}$ and $\mathrm{Bb}$ Plus ELISA kits, according to manufacturer's instructions. Briefly, the assay wells were incubated with wash solution for a duration of one to two minutes at a temperature between 15 and $20^{\circ} \mathrm{C}$ for two to three times. The samples' aliquot of $100 \mu \mathrm{L}$ and standards, in the concentration range from 0 to $237 \mathrm{ng} / \mathrm{mL}(\mathrm{C} 4 \mathrm{~d}$ ) and from 0 to $660 \mathrm{ng} / \mathrm{mL}(\mathrm{Bb})$ were incubated in hydrated micro-assay plates for a period of 30 minutes at room temperature. Afterwards, the wells were washed five times with buffer and $50 \mu \mathrm{L}$ of the appropriate horseradish peroxidase (HRP)conjugated antibodies were added to each test well. The wells were washed with buffer for five times, and $100 \mu \mathrm{L}$ of 
substrate solution was added to each test well. Then, another incubation followed with a duration of 30 minutes (C4d) and 15 minutes $(\mathrm{Bb})$, respectively, was done. After the incubation, the enzyme reaction was stopped chemically by adding $100 \mu \mathrm{L}$ of EDTA stop solution. The optical density of the wells in each kit was measured spectrophotometrically with a plate reader at wavelengths of $405(\mathrm{C} 4 \mathrm{~d})$ and $450 \mathrm{~nm}(\mathrm{Bb})$.

\section{Terminal complement complex (TCC)}

The level of the SC5b-9 complex was evaluated by MicroVue SC5b-9 Plus ELISA kit. The ELISA assay was carried out according to manufacturer's instructions. Briefly, the assay wells were incubated with wash solution for a period of one to two minutes at a temperature between 15 and $20^{\circ} \mathrm{C}$. The samples' aliquot of $100 \mu \mathrm{L}$ and standards, in the concentration range from 0 to $185 \mathrm{ng} / \mathrm{mL}$, were incubated in hydrated micro-assay plates for a duration of 60 minutes at room temperature. After incubation, the wells were washed with buffer for five times and $50 \mu \mathrm{L}$ of HRP-conjugate was added to each test well. After incubation for 30 minutes at room temperature, the wells were washed with buffer for five times, and $100 \mu \mathrm{L}$ of substrate solution was added to each test well. Afterwards, another incubation for a period of 15 minutes took place. Then the enzyme reaction was stopped chemically by adding $100 \mu \mathrm{L}$ of stop solution. The optical density of the wells in each kit was measured spectrophotometrically with a 96-well-plate plate reader (FLUOstar Omega, BMG Labtech, Germany) at wavelength of $450 \mathrm{~nm}$.

\subsection{Statistical analysis}

The levels of SC5b-9, C4d and $\mathrm{Bb}$ in serum are expressed as mean \pm S.D. For data analysis, all samples were compared to saline, except Zymosan. Significance of differences between the groups was determined by ordinary one-way analysis of variance (ANOVA), followed by Dunnett's multiple comparison test. Differences between groups were considered statistically significant at $\mathrm{P} \leq 0.05$. Statistical analysis was carried out using GraphPad Prism 6 (GraphPad Software Inc., CA, USA).

\section{RESULTS}

\subsection{Characteristics of shear-stress-sensitive Pad-PC-Pad liposomes}

\section{Analysis of size and size distribution}

The DLS results indicate the mean diameter of the liposomes containing 5 mol\% DSPE-PEG 2000 corresponded to $115 \mathrm{~nm}$ (B) and $130 \mathrm{~nm}$ (A), while the average size of formulation composed of Pad-PC-Pad only - was above $2.5 \mu \mathrm{m}$ (see Table 1). The liposome stability was tested over 60 days after sample preparation. The related results are shown in Figure 4. Figure 4 A-B demonstrate the presence of only one peak in A and B samples after 60 days, while C shows the presence of three peaks. The two peaks at around 3 and $30 \mu \mathrm{m}$ as well as the broad size distribution reveal the formation of liposomal aggregates. This was reflected in the PDI value, confirming that the sample was poly-disperse.

\section{Analysis of zeta potential}

The zeta potential was in the range from -0.4 to $+3.1 \mathrm{mV}$ (see Table 1). These values indicate that Pad-PC-Pad phospholipids are neutral and should exhibit a relatively low aggregation stability.

Table 1. Properties of liposomal formulations tested in this study. Lipid concentrations of each suspension were determined by the phosphate test 2.0 and shown as the mean of three repeated measurements \pm the relative error. DLS, PDI and $\zeta$ potential values were given as the mean \pm standard deviation of a triplicate measurement.

\begin{tabular}{|c|c|c|c|c|c|c|c|c|c|c|c|c|}
\hline Name & Lipid composition & $\begin{array}{r}\text { Lipid c } \\
(\mathrm{mg} /\end{array}$ & $\begin{array}{l}\text { content } \\
\text { (mL) }\end{array}$ & Averag & siz & $(\mathrm{nm})$ & \multicolumn{3}{|c|}{ PDI } & \multicolumn{3}{|c|}{$\zeta$-potential $(\mathrm{mV})$} \\
\hline A & Pad-PC-Pad/DSPE-PEG 2000 & 8.19 & $\pm \quad 0.95$ & 130.8 & & 3.7 & 0.10 & \pm & 0.01 & -0.42 & \pm & 0.4 \\
\hline B & Pad-PC-Pad/DSPE-PEG 2000 & 9.18 & $\pm \quad 1.15$ & 115.7 & \pm & 0.7 & 0.08 & \pm & 0.01 & +1.26 & & 0.45 \\
\hline $\mathrm{C}$ & Pad-PC-Pad & 10.28 & $\pm \quad 0.40$ & 2659.3 & \pm & 247.4 & 0.91 & \pm & 0.11 & +3.10 & & 0.16 \\
\hline
\end{tabular}


A

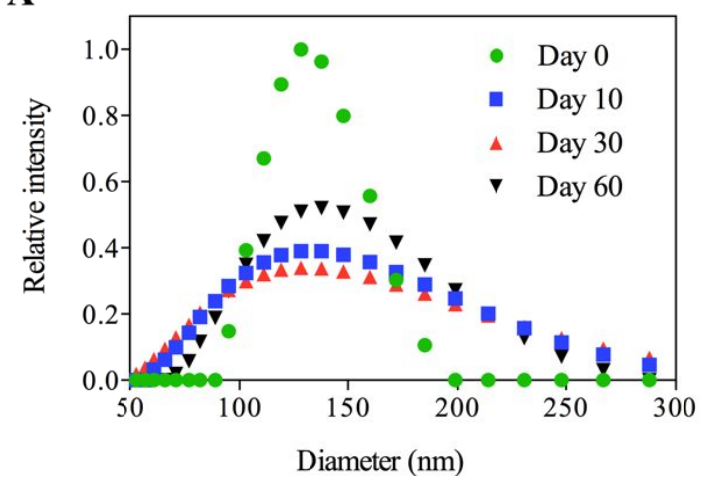

B

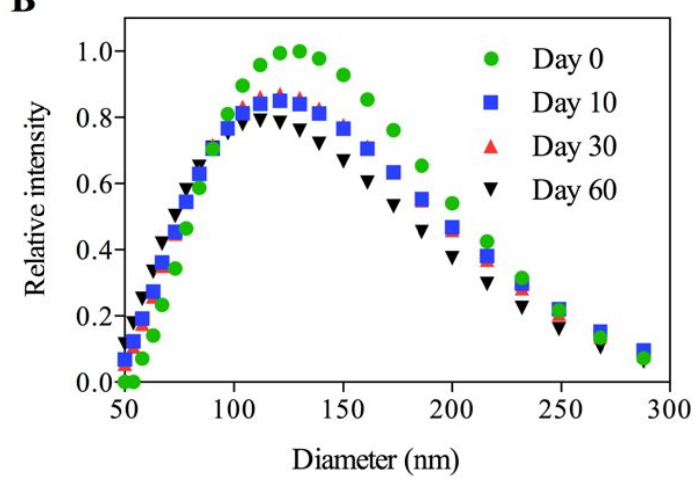

C

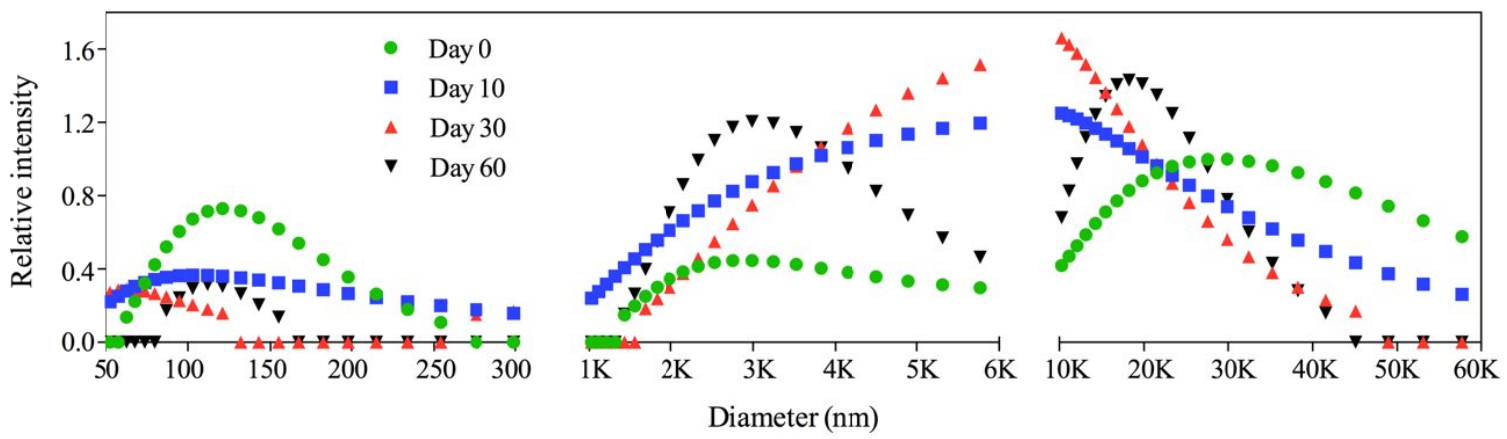

Figure 4. Size distribution of liposome samples A (A), B (B), C (C) measured 0, 10, 30, and 60 days after preparation. Analysis of size and morphology

TEM analysis of all three Pad-PC-Pad liposomal formulation was performed. Figure 5 represents the characteristic micrographs of the A, B, and C samples. The images show a population of heterogeneous liposomes, with mean diameter of $111 \pm 21.1 \mathrm{~nm}$ (A), $106 \pm 23.5 \mathrm{~nm}$ (B) and $102 \pm 17.7 \mathrm{~nm}$ (C). These data corroborate to the results of the DLS analysis (cf. Table 1).
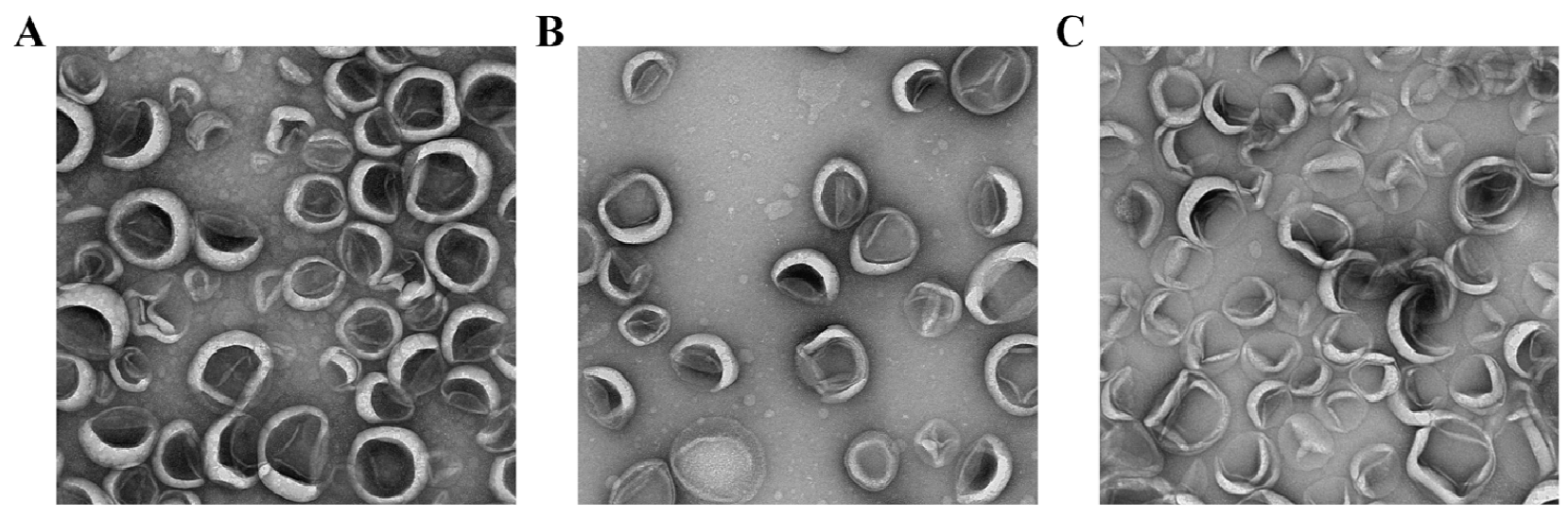

$200 \mathrm{~nm}$

Figure 5. TEM image of liposomal suspensions A (A), B (B), C (C).

\section{Analysis of phospholipid concentration}

The total lipid concentration after extrusion was obtained from the phosphate test 2.0 and found to be in the range between 8.19 and $10.28 \mathrm{mg} / \mathrm{mL}$ (see Table 1). These values were expected, since samples were prepared with an initial 
phospholipid concentration of $10 \mathrm{mg} / \mathrm{mL}$. Both samples A and B contain lower amounts of phospholipids, possibly due to some loss of solution during the preparation steps. When PEG is coupled to hydrophobic molecules (such as phospholipids head groups), it essentially represents non-ionic surfactants. This makes PEGylated samples more difficult to extrude at high pressure without a loss of sample volume. On the contrary, sample $\mathrm{C}$ without PEG has shown a final concentration equivalent within the error to the initial one. This can also happen during the extrusion step. As Pad-PCPad liposomes were extruded at a temperature of $60^{\circ} \mathrm{C}$, after $\sim 25$ extrusion cycles, some volume of loading buffer could evaporate, thus increasing the phospholipid concentration.

\subsection{Encapsulation volume of NTG}

For the liposomal solution with a concentration of $10 \mathrm{mg} / \mathrm{mL}$ and known molecular weight of Pad-PC-Pad $\left(\mathrm{M}_{\mathrm{W}}=734 \mathrm{~g} / \mathrm{mol}\right)$, we calculated the molecular concentration as $0.01 \mathrm{~g}$ of Pad-PC-Pad multiplied by its molecular weight $734 \mathrm{~g} / \mathrm{mol}$ to $1.37 \times 10^{-5} \mathrm{~mol}$. Considering the Avogadro number $6.022 \times 10^{23} \mathrm{~mol}^{-1}$ we calculated the number of Pad-PC-Pad molecules in $10 \mathrm{mg} / \mathrm{mL}$ solution to $8.23 \times 10^{18}$ molecules. We estimated an average liposome with $100 \mathrm{~nm}$ diameter would contain approximately $10^{4}$ Pad-PC-Pad molecules. Therefore, we calculate number of liposomes as number of Pad-PC-Pad molecules $8.23 \times 10^{18}$ divided by $10^{4}$ molecules is equal to $8.23 \times 10^{14}$ liposomes. Based on our previous studies, Pad-PC-Pad liposomes form the lenticular shape. Thus, the volume of one vesicle is calculated to be $4.75 \times 10^{-13} \mu \mathrm{L}$ [7]. Taking this value in consideration, we can estimate the total volume of encapsulated buffer as $8.23 \times 10^{14}$ liposomes times $4.75 \times 10^{-13} \mu \mathrm{L}$, which equals to $391 \mu \mathrm{L}$. The calculated total encapsulation volume for one liposome is therefore $320 \mu \mathrm{L}(\mathrm{A}), 359 \mu \mathrm{L}$ (B), and $402 \mu \mathrm{L}$ (C), respectively.

\subsection{In vitro complement activation for human sera}

\section{Detection of classical and alternative pathways of complement activation}

Figure 6A displays the results of the classical and lectin pathway tests. Formulation B elicited a two-fold elevation in the level of $\mathrm{C} 4 \mathrm{~d}$ versus saline, with a value comparable to Doxil. The level of $\mathrm{C} 4 \mathrm{~d}$ measured in the rest of the samples has not shown any significant difference in comparison to the negative control. Figure $6 \mathrm{~B}$ provides the results of the alternative pathway test. None of the Pad-PC-Pad liposomes, regardless the concentration, showed any significant increase of the $\mathrm{Bb}$ level with respect to saline. $\mathrm{Bb}$ values of the Doxil samples were in the same range as for Pad-PC-Pad liposomes. Abelcet showed more than three-fold increase in $\mathrm{Bb}$ concentration versus saline, and up to eight-fold increase was elicited by Zymosan.

A

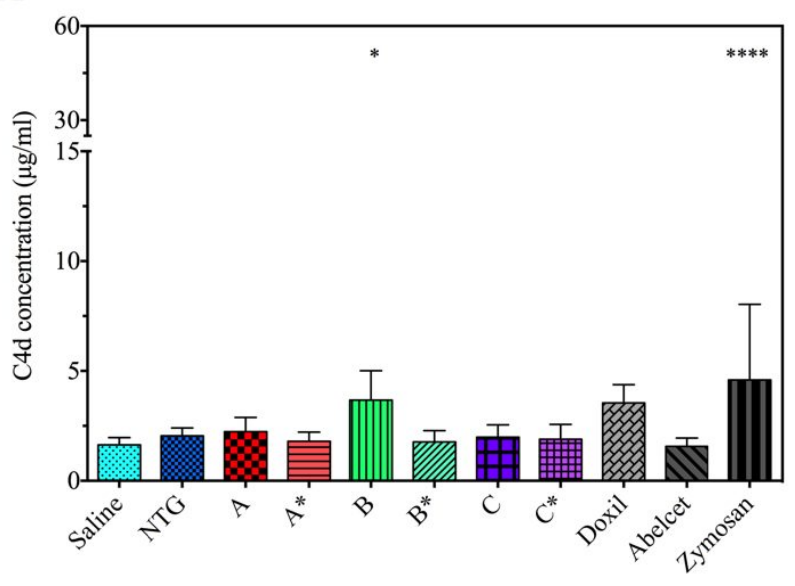

B

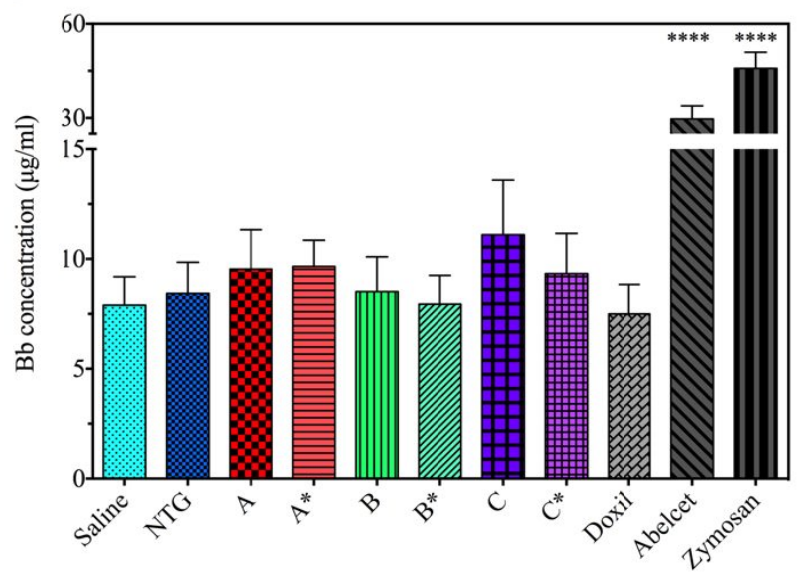

Figure 6. Detection of complement activation via classical, lectin and alternative pathways, using $\mathrm{C} 4 \mathrm{~d}(\mathrm{~A})$ and $\mathrm{Bb}(\mathrm{B})$ ELISA kits. Human sera were incubated for a period of 40 minutes at body temperature with the following samples: saline, nitroglycerin, Pad-PC-Pad liposomes (A, A*, B, B*, C, C*), Doxil, Abelcet and Zymosan. Data are shown as the mean of six sera from donors and error bars as the standard deviation among the individuals. The values were normalized to the liposome concentration of $10 \mathrm{mg} / \mathrm{mL}$ (A, B, C samples) and $1 \mathrm{mg} / \mathrm{mL}$ (A*, B*, C* samples). Significance of differences among the groups was determined by an ordinary one-way ANOVA, followed by Dunnett's multiple comparison test $(\mathrm{P}<0.05)$. 


\section{Detection of terminal complement complex}

Figure 7 demonstrate the results of TCC activation. Formation of SC5b-9 complex was determined and to some extent, all liposomal formulations induced the formation of an SC5b-9 complex. Among the three Pad-PC-Pad liposomal formulations (A, B, C), the sample A, containing Pad-PC-Pad/DSPE- $\mathrm{PEG}_{2000}$ and loaded with nitroglycerin showed the highest SC5b-9 concentration in all tested individuals, 25-fold higher compared to the negative control. Sample C, containing Pad-PC-Pad and loaded with NTG, demonstrated lower, but still elevated level of TCC, that was twelve-fold higher in comparison to saline and NTG. B sample demonstrated the lowest SC5b-9 level that was comparable to Doxil and negative control. Two of the Pad-PC-Pad liposomal formulations, with ten times lower phospholipid concentration $\left(\mathrm{B}^{*}, \mathrm{C}^{*}\right)$ have shown values similar to the negative controls. Within the standard deviation, the results were comparable to Doxil, indicating a mild, biologically non-significant reaction. A* sample displayed four times increased level of SC5b-9 complex, compared to saline and NTG. SC5b-9 concentrations of all three diluted samples were not considered statistically significant $(p>0.05)$, in comparison to saline. The level of SC5b-9 in Abelcet sample was ten-fold higher than in Doxil, and four- to eight-fold higher compared to diluted Pad-PC-Pad samples. Abelcet revealed high complement activation, statistically comparable to that of Zymosan.

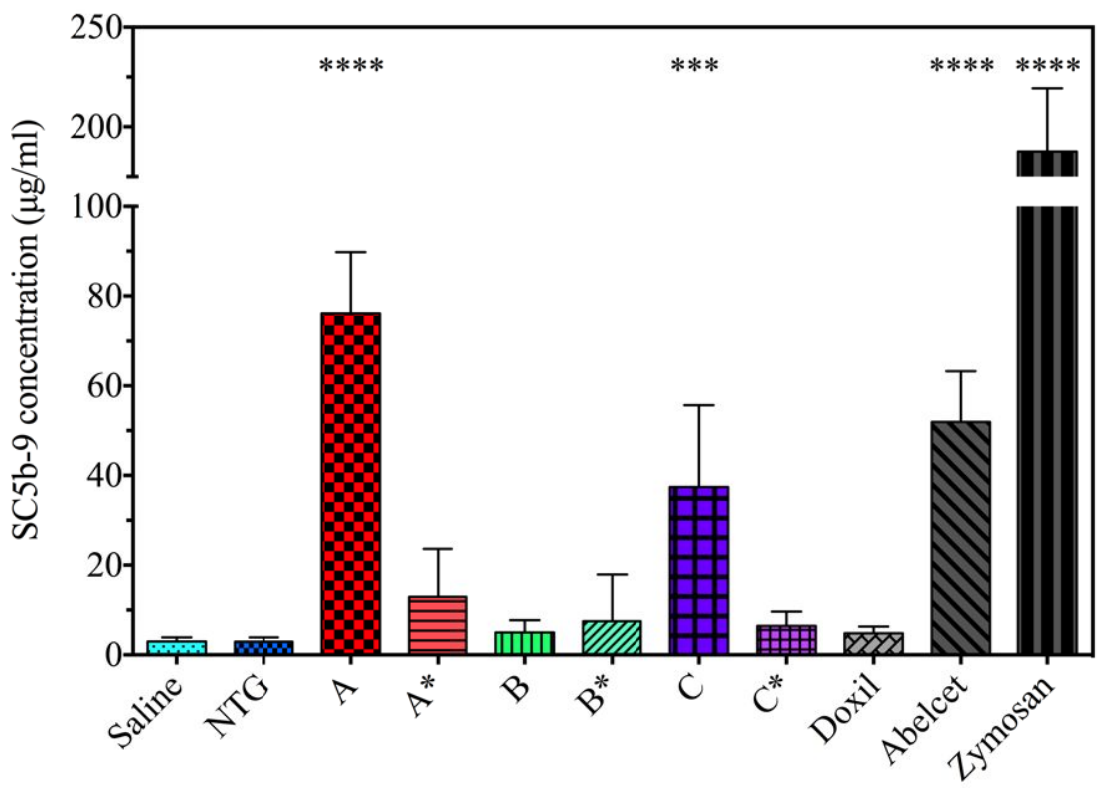

Figure 7. Level of activated SC5b-9 complex, after 40 minutes of incubation at body temperature with human sera. Sera were incubated with saline, nitroglycerin, Pad-PC-Pad liposomes (A, A* B, B*, C, C*), Doxil, Abelcet and Zymosan. Data are shown as the mean of sera from six donors and error bars as the standard deviation among the individuals. The values were normalized to the liposome concentration of $10 \mathrm{mg} / \mathrm{mL}$ (A, B, C samples) and $1 \mathrm{mg} / \mathrm{mL}$ (A*, B*, $\mathrm{C}^{*}$ samples). Significance of differences among the groups was determined by an ordinary one-way ANOVA, followed by Dunnett's multiple comparison test $(\mathrm{P}<0.05)$.

\subsection{Validation of results}

The standard curve of the target protein for each kit is generated using the blank subtracted OD values for each standard and the assigned concentration for each standard (cf. Figure 8). The obtained standard curve must meet the validation requirements. The validation of the ELISA tests results has been verified by the determination of the slope $(\mathrm{m}), \mathrm{y}$ intercept, and correlation coefficient (r) of the derived best-fit for all standards. The results of Bb kits were analyzed using the second-order polynomial model, where instead of slope value, the calibration curve was characterized using the B0, B1 and B2 coefficients. All of the experimental values were within the specified reference ranges according to the kit's manufacturing instructions. Therefore, we can state that obtained results are well reliable and reproducible. 
A

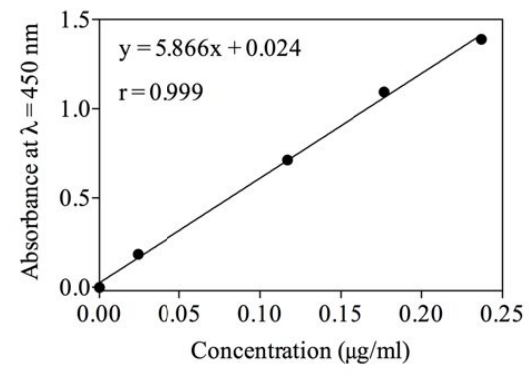

B

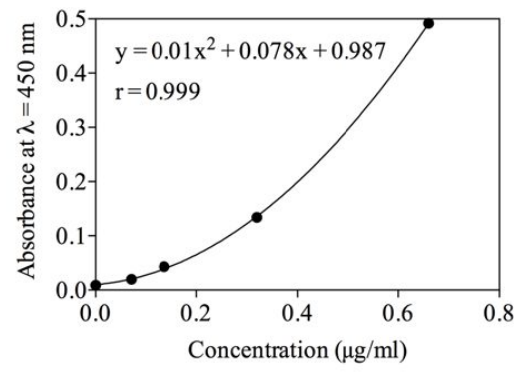

C

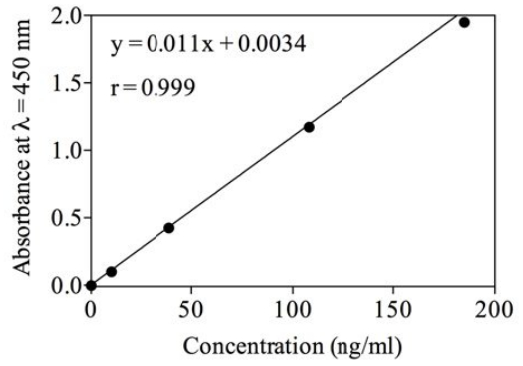

Figure 8. Calibration curves of C4d (A), Bb (B), SC5b-9 (C) ELISA tests.

\section{DISCUSSION}

\section{Pad-PC-Pad liposomal characteristics}

The A and B samples, both containing DSPE- $\mathrm{PEG}_{2000}$ demonstrate average diameter in the range of 115 - $130 \mathrm{~nm}$, while the C sample, composed of Pad-PC-Pad only, has shown a 20 times higher diameter. This indicates the presence of liposomal aggregations within the solution. This observation was confirmed by the analysis of size distribution of the samples on the first day of preparation and after two months (cf. Figure 4C). The sample $\mathrm{C}$ revealed the presence of aggregates immediately after the preparation, and after the rest period of two months. As opposed to $\mathrm{C}$, samples $\mathrm{A}$ and $\mathrm{B}$ were both stable, showed no aggregation and displayed a comparably low PDI value. Low stability of the Pad-PC-Pad sample was predicted by zeta potential measurements: the zeta potential is a key indicator of the stability of the colloidal systems, such as liposomes. It is directly related to the net charge of a particle and affects its mobility in the medium. Zeta potential indicates the degree of electrostatic repulsion between similarly charged particles in the solution. For nanometer-sized particles high zeta potential ensures the stability, meaning the solution will resist aggregation. On the contrary, if the zeta potential is small, attractive forces may dominate, leading to flocculation. The major part of most biological membranes is composed of phospholipids which are zwitterions and therefore neutrally charged. Pad-PC-Pad belongs to this group as well. The polar head group of Pad-PC-Pad molecule contains anionic phosphate and cationic ammonium groups, hence making it a zwitterionic molecule. Carrying both positive and negative electrical charges this molecule reveals a charge, close to neutral. A priori it can be assumed that liposomes with close to zero net charge will possess little or no electrophoretic mobility, therefore, low zeta potentials. This indication was confirmed by zeta potential measurements, where all of the three Pad-PC-Pad samples showed zeta potential close to zero. Colloids with zeta potential $+3 \mathrm{mV}$ are considered as unstable with maximum agglomeration and precipitation [18]. Additionally, the formation of faceted Pad-PC-Pad liposomes hints at very stiff membranes, lacking the undulation forces that lead to vesicle repulsion in standard liposomal formulations [19]. Stable liposome suspensions require repulsive interactions to overcome the attractive forces. Another way to influence liposomes stability is to use steric stabilization, which can be achieved by covering the surface by incorporation of long, bulky molecules into the lipid bilayer. This would prevent aggregation due to increasing the steric distance between liposomes. Polyethylene glycol (PEG) is the most widely used stabilizer in pharmaceutical applications [20] and has been used for liposomes stabilization [21]. Therefore, due to the low stability of pure Pad-PC-Pad sample, we prepared samples containing PEG. Indeed, adding PEGylated lipids into the Pad-PC-Pad vesicles increased the stability of the liposomes and no aggregation was observed, as seen from the DLS results (Figure 4 A, B).

TEM is a powerful high-resolution technique, which may be also applied to characterize the size and morphology of nanoparticles. It allows to produce individual two-dimensional grayscale images, which can be later used to create the three-dimensional structure, using reconstruction software. From the images in Figure 5 it is seen that shape of all three Pad-PC-Pad liposomal samples appeared distorted, showing circular form, and not lentil-shaped, as reported previously [22]. The reason for this lies in the sample preparation where the drying step leads to vesicle rupture. All of the three samples analyzed by TEM revealed lower size when compared, to DLS results. This is related to the liposome shrinkage, caused by sample drying. According to DLS results, sample $\mathrm{C}$ formed aggregates. Nevertheless, according to results of TEM image (Figure 5C), the average size of separated liposomes was $102 \pm 17.7 \mathrm{~nm}$. It is also visible that the liposome distribution on the image is denser in comparison to A and B samples, meaning that pure Pad-PC-Pad liposomes tend to form aggregates. Minor differences in size values between DLS and TEM analysis are present also due to comparatively 
lower count of vesicles analyzed via TEM. It should be noted that TEM analysis is a suboptimal tool to visualize liposomal formulations. In order to preserve initial morphology, and visualize liposomes in their native fully hydrated state, further imaging with a cryo-TEM is desirable.

\section{Human therapeutic dose of NTG}

The dosage of NTG should be adapted to the individual needs of the patient and to the response of controlled parameters, and the respective situation. The clinical dosage range is from 33 to $133 \mu \mathrm{g}$ per minute [23]. Higher doses of $166 \mu \mathrm{g}$ per minute are possible in rare cases [23]. In general, the results of treating acute hypertensive heart failure with intravenous bolus injection of $1 \mathrm{mg} / \mathrm{mL}$ of nitroglycerin were reported [24], as well as patients with severe decompensated heart failure with a bolus injection of $2 \mathrm{mg}$ nitroglycerin [25] has been reported. Based on values obtained with the phosphate test for lipid concentration (Table 1), the encapsulated volume of NTG was calculated (cf. section 3.2). The quantity of nitroglycerin encapsulated in $10 \mathrm{mg} / \mathrm{mL}$ is about $391 \mu \mathrm{g}$, which is three times higher than the maximum dose. Therefore, a ten-fold dilution of each liposomal formulation was prepared and labeled as $\mathrm{A}^{*}, \mathrm{~B}^{*}$ and $\mathrm{C}^{*}$. Phospholipid content and encapsulated volume of NTG for these samples was calculated as ten times lower than initial one. However, the loading procedure of NTG into the liposomes was not optimized and the loading efficiency here certainly lies significantly lower than $100 \%$.

\section{In vitro determination of complement activation}

Overall, the level of $\mathrm{C} 4 \mathrm{~d}$ measured in all samples has shown low concentration of the protein, providing an evidence that complement activation of liposomes does not involve neither classical nor lectin pathways, at least to a major extent. Therefore, as the next step, the complement activation via alternative pathway was studied. We observed elevated levels of $\mathrm{Bb}$ concentration, caused by all the samples. This might be an indication that mainly the alternative pathway is involved in complement activation, caused by liposomal formulation. It was reported that increased $\mathrm{Bb}$ concentration in Doxil-sensitive sera, provides an evidence of its role in alternative pathway activation [26]. Abelcet has shown high activation of $\mathrm{Bb}$ protein, indicating a significant statistical difference. Abelcet is formulated from 1,2-dimyristoyl-snglycero-3-phosphorylcholine (DMPC) and 1,2-dimyristoyl-sn-glycero-3-phosphorylglycerol sodium salt (DMPG), containing amphotericin B, as active drug. A major difference between Abelcet and the rest of the samples is the shape and size of the particles, which could serve as a recognition factor for initiation of the complement activation. It consists of ribbon-like complexes, with size of 1.6 to $11.0 \mu \mathrm{m}$. We expected also high values of protein activation by Zymosan in the $\mathrm{Bb}$ kit, since it is an activator of the alternative pathway. The amount of Zymosan-activated protein increased and multiplied along the activation cascade.

Activation of TCC was represented by the concentration of the soluble C5b-9 protein complex, formed in human sera, after incubation with defined samples. The obtained values were normalized to the liposome concentration of $10 \mathrm{mg} / \mathrm{mL}$ $(\mathrm{A}, \mathrm{B}, \mathrm{C})$ and $1 \mathrm{mg} / \mathrm{mL}\left(\mathrm{A}^{*}, \mathrm{~B}^{*}, \mathrm{C}^{*}\right)$. This step was applied in order to improve data integrity and better differentiate the impact of the liposomal formulation upon the complement activation. Also, this helps to avoid misinterpretation of results due to higher or lower amounts of phospholipids within the sample.

The results of Figure 7 indicate that formulations $\mathrm{A}$ and $\mathrm{C}$ induce relatively strong complement activation. However, some studies suggest that if the complement activation was detected in vitro, does not necessarily mean that a patient will also develop HSRs in vivo. Only those with more than five- to ten-fold increased protein level may carry a risk of complement activation and related HSRs [27]. Such alignment of the results of A and C samples may be caused by the presence of NTG within the vesicles, particularly, by combination of Pad-PC-Pad loaded with NTG. Additionally, under the conditions used, the Pad-PC-Pad vesicles were incubated above their main transition temperature, which should lead to a full release of the entrapped NTG. However, when alone, NTG does not cause detectable immune reaction. Similar situation results were obtained for Doxil, that in the presence of doxorubicin influences the activation of the complement system [26]. Van den Hoven reported that prednisolone sodium phosphate encapsulated within the liposomal aqueous interior also causes increased chance of complement activation [28]. Besides, our recent study demonstrates that drugfree Pad-PC-Pad do not show any significant complement activation [22], as compared to their NTD-loaded liposomal counterparts. As follows, incorporation of certain drugs can cause physicochemical changes to the liposomal formulation, carrying a risk of elevated SC5b-9 concentration. Increased levels of complement activation by C sample can also be correlated with the presence of aggregates, that was confirmed by the DLS measurements. As can be seen from the results in Figure 7, no significant changes in complement activation were seen in the sample B, which contains $\mathrm{PEG}_{2000}$ coupled to DSPE. Sample B showed the lowest value of SC5b-9 concentration among three concentrated samples, and its value was comparable to the negative control. This result was compared with our previously reported 
data, where Pad-PC-Pad liposomes loaded with PBS did not reveal complement activation after a period of 60 minutes of incubation [22]. Van den Hoven also suggests that PEGylated liposomal formulations carry a low risk for causing hypersensitivity reactions [28]. Besides, neither reduction of PEG chain length, nor decrease of PEG density at the liposome surface, significantly changes activation of complement system. However, Chanan-Khan and Szebeni both reported that PEGylated liposomal products, such as Doxil, can induce complement activation-related hypersensitivity reactions $[26,27]$. However, not all the patients with complement activation displayed HSRs. As explained by ChananKhan, it may be related due to the involvement of certain factors, which can limit adverse consequences of activated complement peptides, released during complement activation [27]. The TCC activator Zymosan induces complement activation immediately and after 40 minutes of incubation and has reached up to 62 -fold higher SC5b-9 concentration as compared to saline. It shall be mentioned that for this experiment one donor was found to be consistently more sensitive than the others. This individual could be prone to complement activation and contributes to significantly larger values towards the average (e.g. by factor of two for the $\mathrm{A}^{*}, \mathrm{~B}^{*}, \mathrm{C}^{*}$ ). It is known that all types of liposomes can cause complement activation, which can be even enhanced due to variety of factors, such as positive or negative surface charge, increasing the size of liposomes, PEGylation of liposomes, presence of aggregates, presence of drugs in the extra-liposomal medium, high percentage $(>50 \%)$ of cholesterol in the membrane, etc. [29]. It has been shown that PEGylated liposomes with $120 \mathrm{~nm}$ in diameter, composed of cholesterol, have shown significant increase in activation of $\mathrm{Bb}$, indicating that size and presence of PEG molecules might be important for complement activation by triggering the alternative pathway [28]. Nevertheless, in our study we did not observe the effect of PEGylation on the complement cascade, neither in the pathway, nor in TCC tests. It is important to notice, that the type of molecule used for anchoring the PEG plays a certain role. In our case, we used DSPE, and not cholesterol. However, the liposomal aggregation in the $\mathrm{C}$ sample had its impact on the increased level of TCC formation. Similar statements about direct impact of the liposomal size, charge, and presence of PEG molecules on the complement activation, can be very subjective, as the type of formulation and phospholipid concentration can vary significantly from one liposomal formulation to another. To our knowledge, the complement system reactivity to the synthetic non-natural Pad-PC-Pad liposomes, loaded with nitroglycerin were reported for the first time, therefore a direct comparison with other researcher's reports is complicated.

\section{CONCLUSIONS}

Liposomes can trigger initiation of both classical and alternative complement pathways. Based on the absence of significant changes in $\mathrm{C} 4 \mathrm{~d}$ level, involvement of the classical and lectin pathways can be excluded in the complement activation by Pad-PC-Pad liposomes. Instead, we observed increased levels of $\mathrm{Bb}$ protein, among all the samples that is an indicator of potential involvement of the alternative pathway. However, no statistical significance between our liposomal formulations and the negative control was found. Current preliminary results demonstrate C-activation after 40 minutes of samples' incubation. Further studies will incorporate detailed analysis of complement activation over time, at the various time points. Besides, determination of the SC5b-9 complex formation of NTG-loaded Pad-PC-Pad liposomes in porcine sera would provide additional meaningful information for future in vivo studies.

In summary, our results indicate that nitroglycerin-loaded Pad-PC-Pad liposomes prepared of approximately $10 \mathrm{mg} / \mathrm{mL}$ phospholipids can carry an increased risk on hypersensitivity reaction. Nevertheless, their diluted counterparts demonstrate weak, biologically non-significant, complement activation only in some sensitive donors. Sensitivity for complement activation by liposomes is exhibited on an individual basis, suggesting that sensitive individuals could experience a hypersensitivity reaction. This warns about certain risk for infusion reactions of concentrated Pad-PC-Pad liposomes, loaded with nitroglycerin. However, as mentioned above, diluted liposomal formulations already contain sufficient HTD of the encapsulated drug. Therefore, the application of diluted Pad-PC-Pad liposomes is a promising formulation that deserves to be further investigated.

\section{ACKNOWLEDGMENTS}

This work was primarily funded by the Swiss National Science Foundation (SNSF) in the National Research Program (NRP) 62 'Smart Materials' and supported by the Swiss Government Excellence Scholarships for Foreign Scholars and Artists 2016-2017. The travel award provided by Swiss Society of Biomaterials and Regenerative Medicine is kindly acknowledged. 


\section{REFERENCES}

[1] Evers, P., "Nanotechnology in medical applications: the global market," BCC Research, (2015).

[2] Bangham, A. D., and Horne, R., "Negative staining of phospholipids and their structural modification by surface-active agents as observed in the electron microscope," Journal of molecular biology, 8(5), 660IN2668IN10 (1964).

[3] Felnerova, D., Viret, J.-F., Glück, R., and Moser, C., "Liposomes and virosomes as delivery systems for antigens, nucleic acids and drugs," Current Opinion in Biotechnology, 15(6), 518-529 (2004).

[4] Torchilin, V. P., "Recent advances with liposomes as pharmaceutical carriers," Nature reviews Drug discovery, 4(2), 145-160 (2005).

[5] Gregoriadis, G., "Engineering liposomes for drug delivery: progress and problems," Trends in biotechnology, 13(12), 527-537 (1995).

[6] Fedotenko, I. A., Zaffalon, P.-L., Favarger, F., and Zumbuehl, A., "The synthesis of 1, 3diamidophospholipids," Tetrahedron Letters, 51(41), 5382-5384 (2010).

[7] Holme, M. N., Fedotenko, I. A., Abegg, D., Althaus, J., Babel, L., Favarger, F., Reiter, R., Tanasescu, R., Zaffalon, P.-L., Ziegler, A., Müller, B., Saxer, T., and Zumbuehl, A., "Shear-stress sensitive lenticular vesicles for targeted drug delivery," Nature nanotechnology, 7(8), 536-543 (2012).

[8] Holme, M. N., Schulz, G., Deyhle, H., Weitkamp, T., Beckmann, F., Lobrinus, J. A., Rikhtegar, F., Kurtcuoglu, V., Zanette, I., Saxer, T., and Müller, B., "Complementary X-ray tomography techniques for histology-validated 3D imaging of soft and hard tissues using plaque-containing blood vessels as examples," Nature Protocols, 9(6), 1401-1415 (2014).

[9] WHO, "Cardiovascular disease: heart disease and stroke," Global status report on noncommunicable diseases, 95-103 (2014).

[10] Nossaman, V. E., Nossaman, B. D., and Kadowitz, P. J., "Nitrates and nitrites in the treatment of ischemic cardiac disease," Cardiology in review, 18(4), 190 (2010).

[11] Dawidczyk, C. M., Kim, C., Park, J. H., Russell, L. M., Lee, K. H., Pomper, M. G., and Searson, P. C. "Stateof-the-art in design rules for drug delivery platforms: lessons learned from FDA-approved nanomedicines," Journal of Controlled Release, 187, 133-144 (2014).

[12] Szebeni, J., Muggia, F., Gabizon, A., and Barenholz, Y., “Activation of complement by therapeutic liposomes and other lipid excipient-based therapeutic products: prediction and prevention," Advanced drug delivery reviews, 63(12), 1020-1030 (2011).

[13] Cohen, D., Colvin, R. B., Daha, M. R., Drachenberg, C. B., Haas, M., Nickeleit, V., Salmon, J. E., Sis, B. Zhao, M.-H., "Pros and cons for C4d as a biomarker," Kidney international, 81(7), 628-639 (2012).

[14] Hamad, I., Hunter, A. C., Szebeni, J., Moghimi, S. M., "Poly (ethylene glycol) s generate complement activation products in human serum through increased alternative pathway turnover and a MASP-2-dependent process," Molecular immunology, 46(2), 225-232 (2008).

[15] Walde, P., "Preparation of vesicles (liposomes)," Encyclopedia of nanoscience and nanotechnology, American Scientific Publishers, 8(79), 43-79 (2004).

[16] Olson, F., Hunt, C. A., Szoka, F. C., Vail, W. J., Papahadjopoulos, D., "Preparation of liposomes of defined size distribution by extrusion through polycarbonate membranes," Biochimica et Biophysica Acta (BBA)Biomembranes, 557(1), 9-23 (1979).

[17] Stalder, E., and Zumbuehl, A. "Phosphate Test 2.0," CHIMIA International Journal for Chemistry, 67(11), 819821 (2013).

[18] Riddick, T. M., "Control of colloid stability through zeta potential," Blood, 10(1), (1968).

[19] Sackmann, E. "Membrane bending energy concept of vesicle-and cell-shapes and shape-transitions," FEBS letters, 346(1), 3-16 (1994).

[20] Woodle, M. C., "Sterically stabilized liposome therapeutics," Advanced drug delivery reviews, 16(2), 249-265 (1995).

[21] Immordino, M. L., Dosio F., and Cattel L., "Stealth liposomes: review of the basic science, rationale, and clinical applications, existing and potential," International journal of nanomedicine, 1(3), 297 (2006).

[22] Bugna, S., Buscema, M., Matviykiv, S., Urbanics, R., Weinberger, A., Meszaros, T., Szebeni, J., Zumbuehl, A., Saxer, T., and Müller, B., "Surprising lack of liposome-induced complement activation by artificial 1, 3diamidophospholipids in vitro," Nanomedicine: Nanotechnology, Biology and Medicine, 12(3), 845-849 (2016). 
[23] UCB-Pharma AG, "Perlinganit," [package insert], Bulle, Switzerland (2015).

[24] Wilson, S. S., Kwiatkowski, G. M., Millis, S. R., Purakal, J. D., Mahajan, A. P., and Levy, P. D., "Use of nitroglycerin by bolus prevents intensive care unit admission in patients with acute hypertensive heart failure," The American Journal of Emergency Medicine, 35(1), 126-131 (2017).

[25] Levy, P., Compton, S., Welch, R., Delgado, G., Jennett, A., Penugonda, N., Dunne, R., and Zalenski, R., "Treatment of severe decompensated heart failure with high-dose intravenous nitroglycerin: a feasibility and outcome analysis," Annals of emergency medicine, 50(2), 144-152 (2007).

[26] Szebeni, J., Baranyi, L., Savay, S., Lutz, H. U., Jelezarova, E., Bunger, R., and Alving, C. R., "The role of complement activation in hypersensitivity to pegylated liposomal doxorubicin (Doxil®)," Journal of Liposome Research, 10(4), 467-481 (2000).

[27] Chanan-Khan, A. J., Szebeni, J., Savay, S., Liebes, L., Rafique, N. M., Alving, C. R., and Muggia, F. M., "Complement activation following first exposure to pegylated liposomal doxorubicin (Doxil®): possible role in hypersensitivity reactions," Annals of Oncology, 14(9), 1430-1437 (2003).

[28] Van Den Hoven, J. M., Nemes, R., Metselaar, J. M., Nuijen, B., Beijnen, J. H., Storm, G., and Szebeni, J., "Complement activation by PEGylated liposomes containing prednisolone," European journal of pharmaceutical sciences, 49(2), 265-271 (2013).

[29] Szebeni, J., "Hemocompatibility testing for nanomedicines and biologicals: predictive assays for complement mediated infusion reactions," European Journal of Nanomedicine, 4(1), 33-53 (2012). 